\title{
HIGH RESOLUTION TIME PROFILE OF DECIMETRIC TYPE-III BURSTS
}

\author{
J.R. Cecatto ${ }^{1}$, H.S. Sawant ${ }^{1}$, F.C.R. Fernandes ${ }^{1}$, V. Krishan ${ }^{2}$, J.A.C.F. Neri ${ }^{1}$, and J.C. Moraes Filho ${ }^{1}$ \\ ${ }^{1}$ National Institute of Space Research (INPE), 12201-970, São José dos Campos-SP, Brazil \\ ${ }^{2}$ Indian Institute of Astrophysics, Bangalore, India
}

\begin{abstract}
Type-III bursts are signatures of the electron beams accelerated during the solar flares, their observation and investigation provide information of the acceleration processes, the characteristics of the exciting agent and the acceleration site. The Brazilian Solar Spectroscope (BSS), in operation at INPE, Brazil, have recorded type-III radio bursts in decimetric range (2050-2250 MHz) with high time resolution of $20 \mathrm{~ms}$. Decimetric reverse drift bursts are possibly generated in a dense loop by electron beams travelling towards the photosphere. Hence their time profiles should carry signatures of the density inhomogenities in the loop. Here the temporal and spectral characteristics of decimetric type-III bursts are presented. $\odot 2003$ COSPAR. Published by Elsevier Ltd. All rights reserved.
\end{abstract}

\section{INTRODUCTION}

The decimetric type-III (type-III-dm, hereafter) bursts have been investigated, by various research groups in the frequency range of 1000-3000 MHz (Fernandes, 1992; Sawant et al., 1994; Isliker \& Benz, 1994; Aschwanden et al., 1985; Aschwanden et al., 1995; Fernandes et al., 1996; Melendez et al., 1999), also between 3100-5200 MHz (Stahli \& Benz, 1987), and at $8400 \mathrm{MHz}$ (Benz et al., 1992). However, most of these observations are carried out with time resolution of $100 \mathrm{~ms}$, whereas Melendez et al. (1999) have shown that normally decimetric type-III bursts last for about $300 \mathrm{~ms}$. The observations with a time resolution $\geq 100 \mathrm{~ms}$ are not suitable to make an accurate estimation of their temporal characteristics.

Recently Benz et al. (2001) have reported high sensitivity observations in the $1000-2000 \mathrm{MHz}$ frequency range with a $40 \mathrm{~ms}$ time resolution, whereas Wang et al. (2001) used a $8 \mathrm{~ms}$ time resolution to observe in the range of $2600-3800 \mathrm{MHz}$. However, there is a lack of high time resolution systematic investigations of the time profiles of type-III bursts in the decimetric wavelength range. Preliminary observations of time profiles of the type-III bursts with $20 \mathrm{~ms}$ time resolutions are being reported here for the first time.

\section{OBSERVATIONS AND ANALYSIS}

Brazilian Solar Spectroscope (BSS) (Sawant et al., 2000; 2001) has observed high time resolution (20 ms, 50 $\mathrm{ms}$ ) type-III-dm bursts. Particularly, the observations using a $20 \mathrm{~ms}$ time resolution in 50 frequency channels within the frequency range 2050-2250 MHz were carried out at September, 2001.

Together with the two isolated bursts (observed at 13:03 UT, and 16:06 UT) one group of type-III-dm bursts was observed at 13:04 - 13:05 UT on September 13, 2001. The group consists of more than ten individual type-III-dm bursts. Eight isolated bursts from this group, together the two isolated mentioned above, were selected for detailed analysis of their time profiles. The partial dynamic spectra of the group is shown in Figure 1. Ondrejov Observatory $(0.8-2.0 \mathrm{GHz} ; 2.0-4.5 \mathrm{GHz})$ has also observed these bursts.

From the BSS data we measured the instantaneous bandwidth of each individual type-III burst, and the values ranged from 16 to $188 \mathrm{MHz}$. For $80 \%$ of the bursts the measured instantaneous bandwidth is over $70 \mathrm{MHz}$ with an 
average value of about $100 \mathrm{MHz}$. To estimate the frequency drift rate we considered the time of peak intensity for two distinct frequency channels, in the frequency range of the bursts. The estimated drift rates ranged from 700 to 6400 $\mathrm{MHz} / \mathrm{sec}$. For two cases the drift is over the instrumental limit $\left(10^{4} \mathrm{MHz} / \mathrm{sec}\right.$, for the chosen combination of time resolution, bandwidth, and number of frequency channels). It has to be remarked that reverse drifts were measured for 7 out of the 10 bursts investigated.

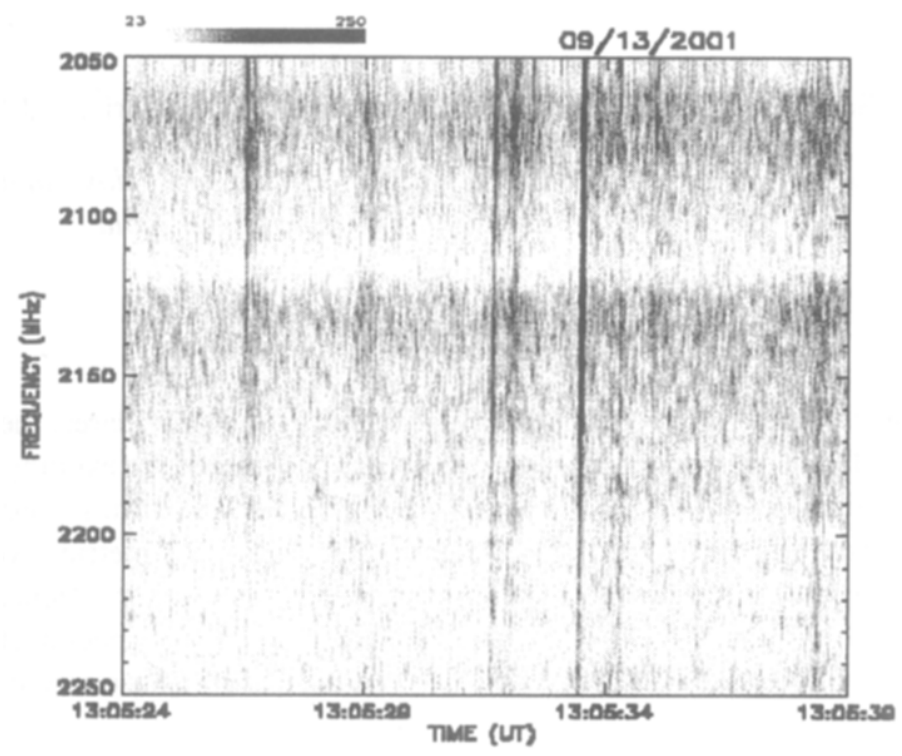

Fig. 1. Dynamic spectra of the high time $(20 \mathrm{~ms})$ resolution type-III-dm bursts observed about 13:05:30 UT on 13th September, 2001.

To obtain the time profiles the background is subtracted and measurements of rise and fall times are carried out at an intermediate frequency channel of the bursts. The time profile of the burst recorded at 13:04:29.6 UT is shown in Figure 2. As seen from the figure, the rise times at half power as well as at the 1/e-fold peak intensity are sligthly shorter than the corresponding decay times of the burst. However, the other burst observed at 13:05:31.9 UT shows a rise time longer than the decay time as seen from Figure 3. Also, bursts are observed with rather symmetric time profiles. The half-power duration in one intermediate frequency channel for the rising phase $\left(\Delta t_{1 / 2 R}\right)$ and the decay phase $\left(\Delta t_{1 / 2}\right)$ along with the e-fall durations $\left(\Delta t_{e} R, \Delta t_{e D}\right)$ for the ten bursts are given in Table 1.

Table I. Properties of High Resolution Type-III Bursts

\begin{tabular}{ccccccccc}
\hline N\# & $\begin{array}{c}\text { Peak time } \\
(\mathrm{UT})\end{array}$ & $\begin{array}{c}\text { Freq. range } \\
(\mathrm{MHz})\end{array}$ & $\begin{array}{c}\text { Instant. } \\
\text { BW (MHz) }\end{array}$ & $\begin{array}{c}\Delta \mathrm{t}_{1 / 2 R} \\
(\mathrm{~ms})\end{array}$ & $\begin{array}{c}\Delta \mathrm{t}_{1 / 2 D} \\
(\mathrm{~ms})\end{array}$ & $\begin{array}{c}\Delta \mathrm{t}_{e R} \\
(\mathrm{~ms})\end{array}$ & $\begin{array}{c}\Delta \mathrm{t}_{e D} \\
(\mathrm{~ms})\end{array}$ & $\begin{array}{c}\text { Drift rate } \\
(\mathrm{MHz} / \mathrm{s})\end{array}$ \\
\hline 1 & $13: 03: 56.9$ & $2050-2250$ & 24 & - & - & - & - & -2690 \\
2 & $13: 04: 29.6$ & $2074-2250$ & 96 & 23 & 31 & 36 & 38 & -2714 \\
3 & $13: 04: 30.8$ & $2218-2234$ & 16 & 18 & 18 & 27 & 28 & $\geq 10^{4}$ \\
4 & $13: 05: 25.1$ & $2050-2138$ & 88 & 19 & 18 & 29 & 26 & $\geq 10^{4}$ \\
5 & $13: 05: 29.8$ & $2050-2206$ & 76 & - & - & - & - & -1767 \\
6 & $13: 05: 31.9$ & $2050-2250$ & 120 & 36 & 24 & 48 & 48 & -2483 \\
7 & $13: 05: 41.0$ & $2050-2250$ & 164 & 77 & 60 & 127 & 207 & -3000 \\
8 & $13: 05: 43.2$ & $2050-2242$ & 76 & 24 & 60 & - & - & -6400 \\
9 & $13: 05: 50.1$ & $2062-2250$ & 188 & 66 & 16 & 81 & 23 & -4857 \\
10 & $16: 06: 57.4$ & $2050-2190$ & 140 & - & - & - & - & 701 \\
\hline
\end{tabular}




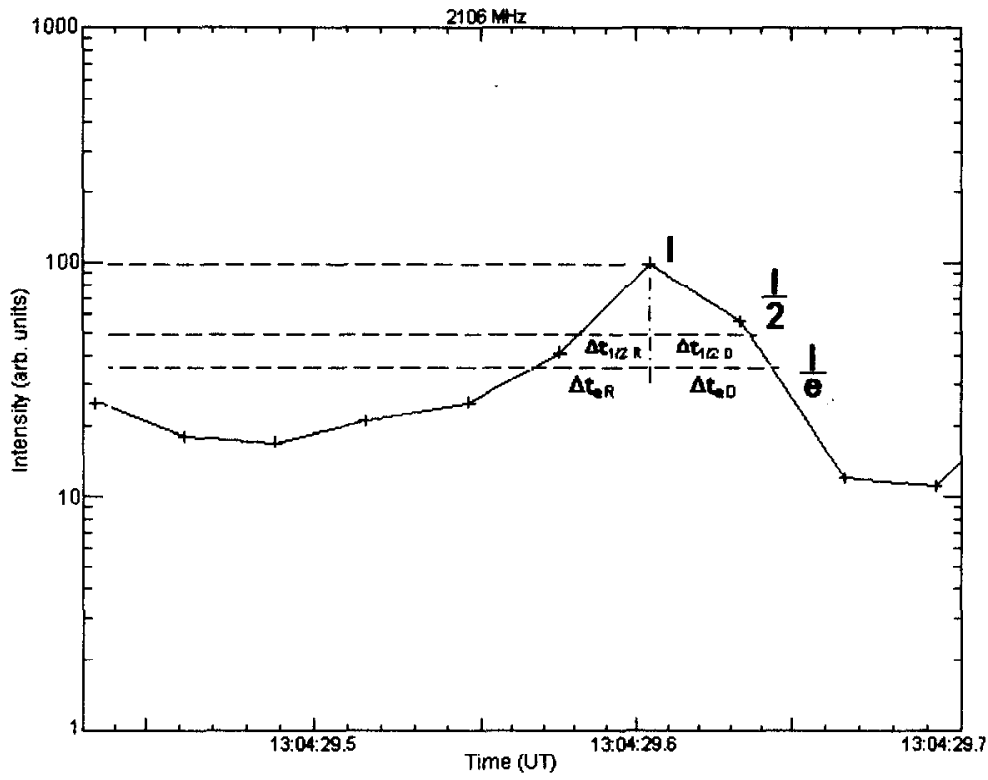

Fig. 2. High resolution (20 ms) time profile at $2106 \mathrm{MHz}$ for the type-III bursts observed about 13:04:29.6 UT on 13th September, 2001. A rise time shorter than the decay time at the half power as well as at 1/e-fold peak intensity is clearly seen.

Also, in the Table are given time of peak intensity, frequency range, instantaneous bandwidth, and frequency drift rate.

For seven bursts the rise times at the half power intensity ranged from 18 to $77 \mathrm{~ms}$ while values within $16-60$ ms were measured for the decay times. For six bursts it was posible to determine the duration at the 1/e-fold peak intensity. The values obtained are within the ranges of $27-127 \mathrm{~ms}$ and $23-207 \mathrm{~ms}$, for the rise and decay phases respectively.

Different fitting functions were tried at two selected frequency channels of the bursts for detailed investigations of the rise and fall time profiles. The results indicate that most, $56 \%$, of the time profiles during the rise phase are fitted by a linear function, although $37 \%$ of them are fitted by an exponential. During the decay phase, the time profiles are dominated by an exponential curve fitting. However, $42 \%$ of time profiles in the decay phase were best fitted by either power-law or linear fitting functions.

\section{INTERPRETATION AND DISCUSSION}

From the meter wave studies, one expects a linear rise and an exponential fall of the radio emission. However, we see from the present observations that this may not be always true for the bursts at decimetric wavelengths. Specially, the high time resolution observations during the rise phase presented here indicate that for 7 of the 10 bursts investigated a linear function is the best fit for the time profile in at least one frequency channel. However, there is a significant percentage of bursts whose rise phase is better fitted by an exponential function. Any departure from the linear rise time is a signature of the nonuniformities in the speed of the electron beam. In fact more than one beam may be responsible for the burst if the rise phase appears to be a complex function of time. The true nature of the velocity distribution and hence the acceleration process of the energetic particles can be studied by obtaining a detailed temporal profile of the rising phase of the bursts.

The exponential fall time of the radio intensity is equal to the inverse of the Coulomb collision frequency between electrons and positive ions $\left(\nu_{e i}\right)$ for the emission at the fundamental and it is equal to four-times this time for the emission at the second harmonic of the electron plasma frequency.

Assuming the type-III-dm emission at second harmonic originates from a region where temperature and density are $10^{6} \mathrm{~K}<T<10^{7} \mathrm{~K} ; N_{e} \simeq 10^{10} \mathrm{~cm}^{-3}$. Then, one can estimate the collision frequency between the electrons 


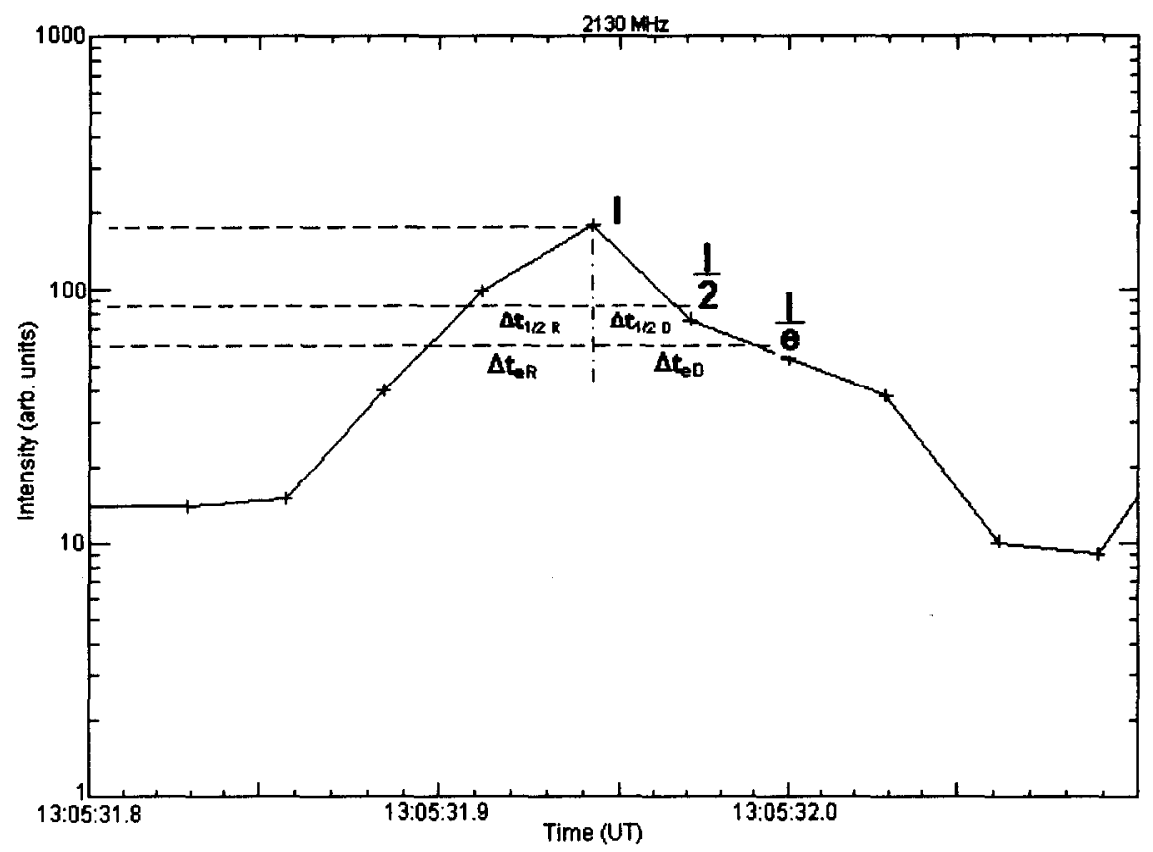

Fig. 3. High resolution ( $20 \mathrm{~ms}$ ) time profile at $2130 \mathrm{MHz}$ for the type-III bursts observed about 13:05:31.9 UT on 13th September, 2001. An asymmetry between the rise and decay times at half power is showed.

and the positive ions. If the relaxation is yielded by the electron-ion Coulomb collisions, then we can estimate the relaxation time approximately as $t_{r} \simeq 4 / \nu_{\text {eff }}$. For the sake of simplicity, we will consider the collisions between electron and ions only. Then, the effective collision frequency of electrons and ions can be given by (Zheleznyakov, 1996):

$$
\nu_{e f f} \simeq \nu_{e i}=\frac{5.5 N_{e}}{T^{3 / 2}} \ln \left(\frac{10^{4} T^{2 / 3}}{N_{e}^{1 / 3}}\right)
$$

where $\mathrm{N}_{e}$ is the electron density, $\nu_{e f f}$, the effective collision frequency, $\nu_{e i}$, the collision frequency between electrons and positive ions, and $\mathrm{T}$, the temperature.

Let $f=2150 \mathrm{MHz}$ be the emission at the second harmonic of the plasma frequency, then $\mathrm{N} \simeq 1.4 \times 10^{10} \mathrm{~cm}^{-3}$. Assuming $\mathrm{T} \simeq 6 \times 10^{6} \mathrm{~K}$, one cstimate $\nu_{\text {eff }} \simeq 16 \mathrm{~s}^{-1}$. In this case, we obtain $\mathrm{t}_{r} \sim 63 \mathrm{~ms}$. This is of the order of the average decay time $\left(\Delta t_{e D}\right)$ we measured from our sample of 10 type-III-dm bursts. Thus, the e-fall times of the bursts reported here can be explained within the Coulomb collision model.

Detailed investigations of the temporal variation of the type-III-dm bursts emission by studying the behavior of the different time durations as a function of the emission frequency, using the 50 frequency channels, as well as the interrelationships if any among the rise and fall durations is under investigation and will be published elsewhere.

\section{CONCLUSIONS}

We have observed type-III-dm radio bursts with high frequency and time resolutions. Investigations of time profile of the type-III bursts with high $(20 \mathrm{~ms})$ time resolution shows that asymmetric and nearly symmetric rise and decay phases are in contrast to the linear rise and exponential decay times as observed in meter wavelengths.

\section{ACKNOWLEDGEMENTS}

Authors acknowledge the financial agencies FAPESP and CNPq that supported BSS. F.C.R.Fernandes and V. Krishan acknowledge receiving scholarship from FAPESP (99/10529-0) and (01/06031-8), respectively. Also, H.S. Sawant acknowledge the grant from FAPESP (01/00056-9) for presentation of this paper in Cospar 2002 Meeting. 
Finally, we are grateful to referees by the comments and suggestions which improved the paper.

\section{REFERENCES}

Aschwanden, M.J., H.J. Wiehl, A.O. Benz et al., Correlation of solar decimetric radio bursts with X-ray flares, Solar Phys., 97, 159-172, 1985.

Aschwanden, M.J., A.O. Benz, B.R. Dennis et al., Solar electron beams detected in hard X-rays and radio waves, Ap.J., 455, 347-365, 1995.

Benz, A.O., A. Magun, W. Stehling et al., Electron beams in the low corona, Solar Phys., 141, 335-346, 1992.

Benz, A.O., P. Messmer, C. Monstein, High-sensitivity observations of solar flare decimeter radiation, Astron. Astrophys., 366, 326-330, 2001.

Fernandes, F.C.R., Dissertação de Mestrado, INPE-5537-TDI/525, DED-INPE, São Jusé dos Campos, SP, Brasil, 1992.

Fernandes, F.C.R., and H.S. Sawant, Physical parameters of the exciter of the decimetric type III bursts, in 6 th Brazilian plasma astrophysics workshop, edited by R.M.O. Galvão, Brazilian Physics Society Series, 4, São José dos Campos, SP, Brasil, pp. 125-128, 1996.

Fürst, E., A.O. Benz, W. Hirth, About the relation between radio and soft X-ray emission in case of very weak solar activity, Astron. Astrophys., 107, 178-185, 1982.

Isliker, H., and A.O. Benz, Catalogue of 1-3 GHz solar flare radio emission, Astron. Astrophys. Suppl. Series, 104, 145-160, 1994.

Kundu, M.R., Solar Radioastronomy, Interscience Publ., New York, USA, 1965.

Melendez, J.L., H.S. Sawant, F.C.R. Fernandes et al., Statistical analysis of high-frequency decimetric type III bursts, Solar Phys., 187, 77-88, 1999.

Riddle, A.C., On the determination of coronal temperature from the decay of type III radio bursts, Solar Phys. 34, 181-184, 1974.

Sawant, H.S., K.R. Subramanian, C. Faria et al., Data acquisition and recent results of the Brazilian Solar Spectroscope - BSS, in HIGH ENERGY SOLAR PHYSICS - Anticipating HESSI, edited by R. Ramaty and N. Mandzhavidze, Astronomical Society of the Pacific Conference Series, 206, pp. 347-350, Sheridan Books Inc., Chelsea, Michigan, 2000.

Sawant, H.S., K.R. Subramanian, C. Faria et al., Brazilian Solar Spectroscope, Solar Phys., 200, 167-176, 2001.

Stähli, M., and A.O. Benz, Microwave emission of solar electron beams, Astron. Astrophys., 175, 271-276, 1987.

Wang, S.J., Y.H. Yan, Q.J. Fu, Group of type I bursts and its associated spikes, Astron. Astrophys., 373, 1083-1088, 2001.

Zheleznyakov, V.V., Radiation in Astrophysical Plasmas, Kluwer Academic Publishers, Dordrecht, Netherlands, 1996.

E-mail address of J.R. Cecatto jrc@das.inpe.br

Manuscript received 01 December, 2002; revised 20 March, 2003; accepted 26 March, 2003 